\title{
University
}

Hoffmann, C. et al. (2017) Persistent anthrax as a major driver of wildlife mortality in a tropical rainforest. Nature, 548(7665), pp. 82-86.

There may be differences between this version and the published version. You are advised to consult the publisher's version if you wish to cite from it.

http://eprints.gla.ac.uk/145915/

Deposited on: 22 August 2017

Enlighten - Research publications by members of the University of Glasgow http://eprints.gla.ac.uk 


\section{Persistent anthrax as a major driver of wildlife mortality in a tropical} rainforest

Authors: Constanze Hoffmann ${ }^{1, \dagger}$, Fee Zimmermann ${ }^{1,2, \dagger}$, Roman Biek ${ }^{3}$, Hjalmar Kuehl ${ }^{4}$, Kathrin Nowak ${ }^{1}$, Roger Mundry ${ }^{4}$, Anthony Agbor ${ }^{4}$, Samuel Angedakin ${ }^{4}$, Mimi Arandjelovic ${ }^{4}$, Anja Blankenburg ${ }^{1}$, Gregory Brazolla ${ }^{4}$, Katherine Corogenes ${ }^{4}$, Emmanuel Couacy-Hymann $^{5}$, Tobias Deschner ${ }^{4}$, Paula Dieguez ${ }^{4}$, Karsten Dierks ${ }^{4}$, Ariane Düx ${ }^{1}$, Susann Dupke ${ }^{2}$, Henk Eshuis ${ }^{4}$, Pierre Formenty ${ }^{6}$, Yisa Ginath Yuh ${ }^{4}$, Annemarie Goedmakers $^{7}$, Jan F. Gogarten ${ }^{1,4,8}$, Anne-Céline Granjon ${ }^{4}$, Scott McGraw ${ }^{9}$, Roland Grunow $^{2}$, John Hart $^{10}$, Sorrel Jones ${ }^{4}$, Jessica Junker ${ }^{4}$, John Kiang ${ }^{11}$, Kevin Langergraber $^{12}$, Juan Lapuente ${ }^{4}$, Kevin Lee ${ }^{4}$, Siv Aina Leendertz ${ }^{1}$, Floraine Léguillon ${ }^{1}$, Vera Leinert ${ }^{13}$, Therese Löhrich ${ }^{1,4}$, Sergio Marrocoli ${ }^{4}$, Kerstin Mätz-Rensing ${ }^{14}$, Amelia Meier $^{4}$, Kevin Merkel ${ }^{1}$, Sonja Metzger ${ }^{1}$, Mizuki Murai ${ }^{4}$, Svenja Niedorf ${ }^{1}$, Hélène De Nys $^{1,4}$, Andreas Sachse ${ }^{1}$, Joost van Schijndel ${ }^{4}$, Ulla Thiesen ${ }^{1}$, Els Ton ${ }^{7}$, Doris Wu ${ }^{1,4}$, Lothar H. Wieler ${ }^{15}$, Christophe Boesch ${ }^{4}$, Silke R. Klee ${ }^{2}$, Roman M. Wittig ${ }^{4}$, Sébastien Calvignac-Spencer ${ }^{1}$, Fabian H. Leendertz ${ }^{1}{ }^{*}$

${ }^{\dagger}$ Equal contributions

*Corresponding author. E-mail: LeendertzF@rki.de

Affiliations:

${ }^{1}$ Robert Koch Institute, P3: "Epidemiology of Highly Pathogenic Microorganisms", Seestraße 10-11, 13353 Berlin, Germany.

${ }^{2}$ Robert Koch Institute, ZBS 2: Centre for Biological Threats and Special Pathogens, Highly Pathogenic Microorganisms, Seestraße 10-11, 13353 Berlin, Germany.

${ }^{3}$ Institute of Biodiversity, Animal Health and Comparative Medicine, Boyd Orr Centre for Population and Ecosystem Health, College of Medical, Veterinary and Life Sciences, University of Glasgow, Glasgow, United Kingdom.

${ }^{4}$ Max Planck Institute for Evolutionary Anthropology (MPI EVAN), Deutscher Platz 6, 04103 Leipzig, Germany.

${ }^{5}$ LANADA/LCVB, Bingerville, 206, Côte d'Ivoire.

${ }^{6}$ World Health Organization, 1211 Geneva 27, Switzerland.

${ }^{7}$ Chimbo Foundation, Amstel 49, 1011 PW Amsterdam, Netherlands.

${ }^{8}$ McGill University, Department of Biology, 855 Sherbrooke Street West Montreal, QC, Canada, H3A 2 T7. 
${ }^{9}$ The Ohio State University, Department of Anthropology, 4034 Smith Laboratory, 174 W. 18th Avenue, Columbus, OH, USA.

${ }^{10}$ Lukuru Foundation, 1235 Avenue des Poids Lourds / Quartier de Kingabois, Kinshasa, DRC.

${ }^{11}$ Limbe Wildlife Centre, Limbe, Cameroon.

${ }^{12}$ Arizona State University, PO Box 872402, Tempe, AZ 85287-2402 USA.

${ }^{13}$ Wild Chimpanzee Foundation (WCF), Deutscher Platz 6, 04103 Leipzig, Germany.

${ }^{14}$ German Primate Center, Kellnerweg 4, 37077 Göttingen, Germany.

${ }^{15}$ Robert Koch Institute, Seestraße 10-11, 13353 Berlin, Germany.

Anthrax is a globally significant animal disease and zoonosis. Despite this, current knowledge of anthrax ecology is largely limited to arid ecosystems, where outbreaks are most commonly reported ${ }^{1-3}$. We reveal the dynamics of an anthrax causing agent, Bacillus cereus biovar anthracis, in a tropical rainforest with severe consequences for local wildlife communities. Using data and samples collected over three decades we find that rainforest anthrax is a persistent and widespread cause of death for a broad range of mammalian hosts. We predict that this pathogen will accelerate the decline and possibly result in the extirpation of local chimpanzee (Pan troglodytes verus) populations. Our findings illuminate the epidemiology of a cryptic pathogen and have important implications for conservation.

Anthrax is a disease of wildlife, livestock and humans predominantly affecting low and middle-income countries ${ }^{2,4,5}$. Although widely distributed, including some temperate regions, anthrax is most commonly associated with arid ecosystems, particularly African 
71 savannas ${ }^{1,3,6-11}$. In these systems, major outbreaks typically cause high mortality in a few

72 wild and domestic ungulate species at a time and usually exhibit strong seasonal and

73 inter-annual variation ${ }^{2,3,5,11,12}$. For example, in Krüger National Park, South Africa, die-

74 offs in kudus (Tragelaphus strepsiceros) and impalas (Aepyceros melampus) occur in the

75 dry season with a ten year periodicity coinciding with rainfall cycles ${ }^{11}$. In Etosha

76 National Park, Namibia, mortality in elephants (Loxodonta Africana) peaks at the start of

77 the wet season, while plains ungulates (Equus quagga, Conochaetes taurineus,

78 Antidorcas marsupialis) are most affected at the end of the wet season ${ }^{3,13}$. Such varying

79 dynamics underline the importance of investigating the pathogen in close relation with its

80 ecosystem, but so far anthrax research in Africa has been biased towards well-studied

81 savanna regions.

82 In 2001, lethal anthrax-like cases in wild chimpanzees were reported in a rainforest

83 habitat: Taï National Park (TNP), Côte d'Ivoire (Fig. S1) ${ }^{14}$. The causative agent was a

84 bacterium combining the chromosomal background of Bacillus cereus with the virulence

85 plasmids of $B$. anthracis (Bacillus cereus biovar anthracis; Bcbva) ${ }^{15}$. Pathology and

86 histopathology of Bcbva cases were clearly suggestive of anthrax and in small animal

87 models Bcbva was as virulent as B. anthracis ${ }^{14-16}$. Bcbva cases have since been described

88 in animals in Cameroon (CM), Central African Republic (CAR) and the Democratic

89 Republic of Congo ${ }^{17,18}$, suggesting a broad sub-Saharan distribution (Fig. 1). However,

90 the epidemiology of anthrax-like disease caused by Bcbva (hereafter anthrax), and to

91 what extent it matches that of classical anthrax, remain poorly understood.

92 We address this knowledge gap by testing a unique set of samples collected in TNP over

9326 years. We started collecting bones in 1989 resulting in bones from 75 individual 
mammals (Table S7, Supplementary information S4). From 1996 on, we investigated 204 fresh carcasses (Table S2, Supplementary information S2). Since bone and carcass discovery was linked to the collection of chimpanzee behavioral data, we expected detection of $B c b v a$ to be biased towards chimpanzees and other easily detectable medium to large-bodied mammals. We therefore tested whether carrion flies, which are relatively unbiased samplers of mammalian DNA ${ }^{19}$, might also collect $B c b v a$ or its genetic material while feeding and ovipositing on carcasses. Starting in 2008, we applied different horizontal and vertical sampling schemes to collect 1,634 flies (Table S1 and S4, Supplementary information S3). We retrieved Bcbva isolates from all three sample types (bones, carcasses, flies). These allowed us to generate 178 whole genome sequences spanning from 1996 to 2014 (Table S8). To clarify the distribution of Bcbva on a larger scale, we sampled 1089 flies and 136 bones from 16 other sites in 11 sub-Saharan countries from 2012 to 2014 (Fig. 1, Table S1).

In TNP we detected Bcbva DNA in 81 carcasses (40\%; Fig. 2A, Extended Data Fig. S1, Extended Data Fig. S2, Table S2), 26 bones (35\%, Table S7) and 80 flies (5\%; Fig. 2B, Extended Data Fig. S3, Table S4). We could perform histopathological examinations on 15 positive carcasses and in all cases pathology was consistent with a lethal anthrax infection (Table S2). Overall, 38\% of observed local wildlife mortality was associated with Bcbva (Tables S2 and S4), meeting the highest levels of mortality reported for classical anthrax outbreaks in savanna ecosystems ${ }^{12,20}$. We observed no obvious seasonal variation in Bcbva carcass incidence, suggesting ongoing anthrax activity in the area (Generalized Linear Mixed Model (GLMM), $\chi 2=6.3, \mathrm{df}=10, \mathrm{P}=0.789$, Supplementary information S8a). However, Bcbva detection in flies peaked from December to March, 
117 coinciding with the only distinct dry period in the park (GLMM, $\chi 2=6.9, \mathrm{df}=2, \mathrm{P}=0.032$,

118 Extended Data Fig. S4, Supplementary information S8b). This suggests climatic

119 conditions may influence $B c b v a$ ecology in TNP, similar to observations from $B$.

120 anthracis in savannas ${ }^{1}$, though seasonal mortality appears less pronounced.

121 Bcbva differed dramatically from B. anthracis in terms of host range. Ungulates

122 constitute the vast majority ( $>99 \%$ ) of anthrax cases in savanna ecosystems ${ }^{11,12,20}$. In

123 contrast, and in line with the more diverse fauna found in rainforests, we observed $B c b v a$

124 fatalities in a broader range of species in TNP, including chimpanzees (31/55), six

125 monkey species (21/81), duikers (26/40), mongooses (2/2) and porcupines (1/26 other

126 mammals) (Table S2). To further explore the host range of Bcbva, we analyzed the gut

127 content of all mammal and $B c b v a$ positive flies $(\mathrm{n}=28$, Table $\mathrm{S} 1)$ using amplicon deep

128 sequencing. We detected sequences from most of the aforementioned species, and from

129 species belonging to 11 further mammalian genera, including carnivores, rodents and bats

130 (Table S5, Supplementary information S3e). This suggests that Bcbva may affect an even

131 broader range of mammals than inferred from carcass monitoring alone. Further, meal

132 compositions of mammal positive $B c b v a$ positive flies $(\mathrm{n}=28)$ and mammal positive

133 Bcbva negative flies $(\mathrm{n}=29)$ did not differ significantly (GLMMs, Supplementary

134 information S8c), which may support the notion that there is no substantial difference in

135 Bcbva susceptibility among species.

136 To gain further insight into the ecology of $B c b v a$, we investigated 178 genomes derived

137 from isolates obtained from necropsy samples, bones and flies, collected between 1996

138 and 2014 (Table S8). Considering 126 chromosomal sequences originating from separate

139 hosts (mammals and flies) we detected 298 single nucleotide polymorphisms (SNP). 
140 Plasmids contained negligible amounts of variation (Supplementary information S7a).

141 The maximum distance observed between isolates was 69 SNPs (median: 26 SNPs); the

142 most distant isolates originated in flies caught in two consecutive years only $6 \mathrm{~km}$ apart.

143 In comparison, a maximum distance of only 20 SNPs was observed in B. anthracis

144 isolates derived from cattle samples collected in the French Alps between 1997 and

$1452009^{21}$. The high genetic diversity observed in TNP is consistent with extensive Bcbva

146 activity in the area and suggests that this pathogen did not emerge recently (Fig.3,

147 Extended Data Fig. S5). In addition, considerably more divergence was seen compared to

148 isolates from other countries ${ }^{17,18}$, supporting the notion that $B c b v a$ has been circulating in

149 sub-Saharan Africa for an even much longer period than what we determined in TNP

150 (Extended Data Fig. S6, Supplementary information S7).To assess within-host diversity

151 we sequenced the genomes of two to six independent isolates for a subset of carcasses

152 and flies (Table S9). Two strains differing by 42 chromosomal SNPs were isolated from a

153 single fly, likely reflecting multiple carcass meals ${ }^{19}$, which further highlights the

154 commonness of Bcbva in TNP. Otherwise, the maximum distance observed within one

155 host was two chromosomal SNPs (mean: 0.35 SNPs). Within-host heterogeneity thus

156 seems negligible compared to the overall diversity observed for Bcbva suggesting strains

157 differing by more than two SNPs originate from separate carcasses.

158 Bcbva positive carcasses were broadly distributed throughout the TNP research area,

159 without the kind of geographic clustering described for anthrax in savanna

160 ecosystems $^{12,22}$ (Fig. 2A). We determined Bcbva prevalence within and outside the

161 research area using a subset of 908 flies caught systematically according a grid system

162 within 19 days (Extended Data Fig. S7). We detected Bcbva positive flies in 16/83 traps 
163 (Additional Data Table S1). Prevalence was higher in the research area (8/21 traps Bcbva

164 positive) than in the surrounding forest belt (8/62 traps Bcbva positive) (Fisher's Exact

165 Test, $P=0.02)$. Long-term research activity within the TNP research area has had a

166 protective effect on wildlife and led to an increased density of mammals ${ }^{23}$, which might

167 explain higher $B c b v a$ activity. Genome data revealed multiple contemporaneous

168 transmission chains caused by co-circulating strains (2 to 48 SNPs distance, median: 25

169 SNPs) in different areas of the park over the short time period of the fly snapshot

170 (Extended Data Fig. S8). For low genomic distances ( $\leq 35$ SNPs), genomic and

171 geographic distances of all TNP isolates were positively correlated $\left(\mathrm{R}^{2}=0.72\right)$, providing

172 further indication of spatially restricted transmission (Extended Data Fig. S9), which

173 might reflect carcass-mediated spread of Bcbva. Since wildlife cases included exclusively

174 arboreal monkeys (Table S2), we explored the vertical distribution of Bcbva by catching

175 flies simultaneously on the ground and up to $30 \mathrm{~m}$ into the canopy. We detected Bcbva in

17612 of 103 canopy flies (11.7\%) and retrieved isolates from five of these (Table S4,

177 Additional Data Table S1). While on the ground carcass deposition sites are likely to be

178 the source of $B c b v a$ infections, flies may contribute to Bcbva transmission in the upper

179 strata of the rainforest ${ }^{24}$.

180 Fly samples indicated a large proportion of undetected anthrax mortality. During 19 days

181 of focused fly sampling, we retrieved $B c b v a$ isolates from 17 flies, with 13 strains being

182 more than two SNPs different from any other strain. Since two SNPs appear to be the

183 upper level of within-host diversity (Table S9), this implies the presence of at least 13

184 different Bcbva positive carcasses. Yet, during the same sampling period, only three

$185 B c b v a$ positive carcasses were discovered and their isolates all corresponded to one of the 
186 fly Bcbva lineages ( $\leq 2$ SNPs difference). This suggests carcass monitoring alone

187 underestimates mortality by at least an order of magnitude.

188 We investigated the consequences of $B c b v a$-induced mortality on the species best studied

189 in this ecosystem, chimpanzees. Chimpanzees have a low reproduction rate ${ }^{25}$ and are thus

190 particularly sensitive towards external changes to their environment. Based on

191 demographic data collected from habituated groups in TNP, we simulated population

192 viability at a 150 years horizon across a broad range of demographic models including

193 and excluding anthrax induced mortality (Fig. S7 and S8). Our simulations showed that,

194 with $B c b v a$, the TNP chimpanzee population would only have high chances to persist in

195 the case of an overall annual per capita mortality rate due to other causes of $1 \%$ (Fig. S7

196 and S8). Such a low mortality rate is, however, not even observed in captive

197 chimpanzees. In wild chimpanzees the lowest annual per capita mortality rate is $4 \%$ (in

198 early adults) ${ }^{25}$. Under such a survival probability (0.96), the simulated presence of

199 anthrax invariably led to a clearly reduced survival probability of communities (Fig. 4).

200 For example, $76 / 84$ models resulted in extirpation probability higher than $50 \%$, while the

201 model which we consider the most realistic (community size 60, maximum age 46 years

202 and inter birth interval 6 years) resulted in an extirpation probability of $89 \%$ (Fig. 4). Our

203 simulations therefore suggest that anthrax induced mortality will result in deterministic

204 population declines and possible extirpation of TNP chimpanzees over the next 150

205 years. The risk of extirpation will increase if chimpanzee mortality due to hunting and

206 human-borne infectious diseases continues to rise ${ }^{23,26,27}$.

207 To determine whether similar unrecognized effects on wildlife might be occurring

208 elsewhere, we tested 784 flies collected at eight different sites, as well as 136 bones from 
twelve sites in five and nine sub-Saharan countries, respectively (Fig. S3, Table S1). All sites had chimpanzee populations but none (nor the country) had previously reported Bcbva cases. We only detected Bcbva genetic material in 2 of 105 flies and 1 of 8 bones collected in the Grebo National Forest (GNF) in Liberia, about $40 \mathrm{~km}$ from TNP (Fig.

$\mathrm{S} 1)$. The genome sequences of isolates from the two fly samples nested within the diversity of Bcbva in TNP which may indicate an epidemiological link (Fig. 3). We did not detect $B c b v a$ in 305 flies from two sites where $B c b v a$ cases have been previously reported (Dja Reserve, CM, and Dzanga Sangha Protected Areas, CAR, Table S4). While the lack of detection at other sites needs to be interpreted with caution due to variable fly species composition (Extended Data Fig. S10, Supplementary information S3f), these data suggest that $B c b v a$ dynamics may also vary across rainforest ecosystems. It will be important to further uncover the scale and environmental drivers behind Bcbva prevalence. Such knowledge will be critical for mitigating against the detrimental effects of Bcbva on wildlife and for better assessing human infection risk, which for anthrax in rainforest ecosystems has, to date, been considered very low.

\section{References (1-27)}

1 Hampson, K. et al. Predictability of anthrax infection in the Serengeti, Tanzania. $J$ Appl Ecol 48, 1333-1344, doi:10.1111/j.1365-2664.2011.02030.x (2011).

2 Hugh-Jones, M. \& De Vos, V. Anthrax and wildlife. Revue Scientifique et Technique-Office International des Epizooties 21, 359-384 (2002).

3 Lindeque, P. M. \& Turnbull, P. C. Ecology and epidemiology of anthrax in the Etosha National Park, Namibia. Onderstepoort J Vet Res 61, 71-83 (1994).

4 Beyer, W. \& Turnbull, P. C. Anthrax in animals. Mol Aspects Med 30, 481-489, doi:10.1016/j.mam.2009.08.004 (2009).

5 Turnbull, P. C. B. WHO Guidelines Approved by the Guidelines Review Committee (World Health Organization, Department of Communicable Diseases Surveillance and Response, 2008). 
2376 Good, K. M., Houser, A., Arntzen, L. \& Turnbull, P. C. Naturally acquired

238 anthrax antibodies in a cheetah (Acinonyx jubatus) in Botswana. Journal of

$239 \quad$ wildlife diseases 44, 721-723 (2008).

2407 Wafula, M. M., Patrick, A. \& Charles, T. Managing the 2004/05 anthrax outbreak

241 in Queen Elizabeth and Lake Mburo National Parks, Uganda. African Journal of

242 Ecology 46, 24-31 (2008).

2438 Clegg, S., Turnbull, P., Foggin, C. \& Lindeque, P. Massive outbreak of anthrax in

244

245

246

247

248

249

250

251

252

253

254

255

256

257

258

259

260

261

262

263

264

265

266

267

268

269

270

271

272

273

274

275

276

277

278

$279 \quad 22$

280 wildlife in the Malilangwe Wildlife Reserve, Zimbabwe. The Veterinary Record 160, 113-118 (2007).

9 Muoria, P. K. et al. Anthrax outbreak among Grevy's zebra (Equus grevyi) in Samburu, Kenya. African Journal of Ecology 45, 483-489 (2007).

10 Turnbull, P. et al. Anthrax in wildlife in the Luangwa Valley, Zambia. The Veterinary Record 128, 399-403 (1991).

11 de Vos, V. The ecology of anthrax in the Kruger National Park, South Africa. Salisbury Med Bull Suppl 68, 19-23 (1990).

12 Lembo, T. et al. Serologic surveillance of anthrax in the Serengeti ecosystem, Tanzania, 1996-2009. Emerg Infect Dis 17, 387-394, doi:10.3201/eid1703.101290 (2011).

13 Beyer, W. et al. Distribution and molecular evolution of Bacillus anthracis genotypes in Namibia. PLoS neglected tropical diseases 6, e1534 (2012).

14 Leendertz, F. H. et al. Anthrax kills wild chimpanzees in a tropical rainforest. Nature 430, 451-452, doi:10.1038/nature02722 (2004).

15 Klee, S. R. et al. The genome of a Bacillus isolate causing anthrax in chimpanzees combines chromosomal properties of $B$. cereus with $B$. anthracis virulence plasmids. PLoS One 5, 0010986 (2010).

16 Brézillon, C. et al. Capsules, toxins and AtxA as virulence factors of emerging Bacillus cereus biovar anthracis. PLoS Negl Trop Dis 9, e0003455, doi:10.1371/journal.pntd.0003455 (2015).

17 Antonation, K. S. et al. Bacillus cereus biovar anthracis causing anthrax in subSaharan Africa - Chromosomal monophyly and broad geographic distribution. PLOS Neglected Tropical Diseases (2016).

18 Leendertz, F. H. et al. Anthrax in Western and Central African great apes. American journal of primatology 68, 928-933, doi:10.1002/ajp.20298 (2006).

19 Calvignac-Spencer, S. et al. Carrion fly-derived DNA as a tool for comprehensive and cost-effective assessment of mammalian biodiversity. Molecular ecology 22, 915-924, doi:10.1111/mec.12183 (2013).

20 Berry, H. H. Surveillance and control of anthrax and rabies in wild herbivores and carnivores in Namibia. Rev Sci Tech 12, 137-146 (1993).

21 Vergnaud, G. et al. Comparison of French and worldwide Bacillus anthracis strains favors a recent, Post-Columbian origin of the predominant NorthAmerican Clade. PLoS One 11, e0146216, doi:10.1371/journal.pone.0146216 (2016).

22 Smith, K. L. et al. Bacillus anthracis diversity in Kruger National Park. Journal of clinical microbiology 38, 3780-3784 (2000). 
23 Campbell, G., Kuehl, H., Diarrassouba, A., N'Goran, P. K. \& Boesch, C. Longterm research sites as refugia for threatened and over-harvested species. Biology letters 7, 723-726, doi:10.1098/rsbl.2011.0155 (2011).

24 Blackburn, J. K., Van Ert, M., Mullins, J. C., Hadfield, T. L. \& Hugh-Jones, M. E. The necrophagous fly anthrax transmission pathway: empirical and genetic evidence from wildlife epizootics. Vector-Borne and Zoonotic Diseases 14, 576583 (2014).

25 Hill, K. et al. Mortality rates among wild chimpanzees. Journal of human evolution 40, 437-450, doi:10.1006/jhev.2001.0469 (2001).

26 Köndgen, S. et al. Pandemic human viruses cause decline of endangered great apes. Current biology : $C B$ 18, 260-264, doi:10.1016/j.cub.2008.01.012 (2008).

27 Boesch, C. \& Boesch-Achermann, H. The chimpanzees of the Taï Forest: Behavioural ecology and evolution. (Oxford University Press, USA, 2000).

\section{Supplementary information}

Supplementary information: this file contains a more detailed method section as well as additional tables (Tables S1-10) and figures (Fig. S1-8).

Additional Data Table S1: Results derived from the analyses of flies caught in TNP analyzed in this study. This file includes results from PCR and culture as well as flymeal analysis results for a selection of flies.

Additional Data Table S2: Results of fly meal analysis with taxonomic assignment at genus level. This file provides the number of sequences per amplicon assigned at genus level.

Additional Data Table S3: Results of fly meal analysis with taxonomic assignment at order level. This file provides the number of sequences per amplicon assigned at order level.

\section{Acknowledgements}

We thank the authorities in Côte d'Ivoire for long-term support, especially the Ministry of the Environment and Forests, the Ministry of Research, the directorship of the Taï 
311 National Park, and the CSRS in Abidjan. We also thank the national authorities from all

312 other countries for providing permissions for our research (MINFoF, MINRESI, the

313 Service de la Conservation de la Réserve du Dja, Cameron, in CAR the Ministère d'Eaux

314 et Fôret, Chasse et Peche and the Ministère de l'Education Nationale, de

315 l'Alphabetisation, de l'Enseignement Superieur, et de la Recherche, the Agence

316 Nationale des Parcs Nationaux, Gabon, Centre National de la Recherche Scientifique et

317 Technologique, Gabon, Direction des Eaux, Forêts et Chasses, Senegal, Forestry

318 Development Authority, Liberia, Institut Congolais pour la Conservation de la Nature,

319 DR-Congo, Ministère de l'Agriculture de l'Elevage et des Eaux et Forêts, Guinea,

320 Instituto da Biodiversidade e das Áreas Protegidas (IBAP), Guinea-Bissau, Ministère de

321 la Recherche Scientifique, DR-Congo, Ministère de le Recherche Scientifique et

322 Technologique, R-Congo, Nigeria National Park Service, Nigeria, Uganda National

323 Council for Science and Technology, Ugandan Wildlife Authority, Uganda).

324 We thank the WWF CAR for their logistical support. For the collection of samples in

325 TNP we thank the field assistants, Arthur Henlin, Katerina Albrechtova and Alexander

326 Lang. We are also grateful to the field assistants from all other sites for their support. For

327 laboratory we are grateful to Silke Becker, Tatjana Franz, Sabine Howaldt, Angelika

328 Lander, Petra Lochau, Herbert Nattermann and Andy Schneider, for sequencing we thank

329 the RKI Central Sequencing Laboratory, particularly Julia Hinzmann, Andreas Nitsche

330 and Julia Tesch and for bioinformatic support to Piotr Wojciech Dabrowski and Torsten

331 Semmler from RKI, as well as Graham Hamilton at Glasgow Polyomics. We would

332 further like to thank Teresa Börding, Thurston Hicks, Yasmin Moebius, Volker Sommer,

333 Klaus Zuberbühler and Martine Peeters, and for administrative support Maja Kovacev- 
334 Wegener. For funding we thank the German Research Council DFG KL 2521/1-1 and the 335 Sonnenfeld-Stiftung. We would like to thank the Max-Planck-Society and Krekeler 336 Foundation for funding of the Pan African Programme.

\section{Author contributions}

CH, FZ, AA, SA, MA, GB, KC, PD, KD, HE, PF, YG, AG, AG, SMG, JH, SJ, JJ, JK, according field data. Necropsies on wildlife found dead were performed by FZ, KN, AB, ECH, AD, PF, SAL, TL, SM, SN, HDN and FHL and laboratory analyses performed by analyzed by $\mathrm{CH}, \mathrm{FZ}, \mathrm{RB}, \mathrm{HK}, \mathrm{RM}$ and $\mathrm{SC}$ and the manuscript prepared by $\mathrm{CH}, \mathrm{FZ}, \mathrm{RB}$, HK, RM, JG, SC and FHL. The manuscript was revised and approved by all authors. The

346 study was supervised by CB, RW, SC and FHL.

\section{Author information}

349 Reprints and permissions information is available at www.nature.com/reprints. The

350 authors declare no competing financial interests. Correspondence and requests for 351 materials should be addressed to leendertzf@,rki.de.

\section{$353 \quad$ Figure legends}

354 Fig. 1. Bcbva occurrence and study sampling sites in sub-Saharan Africa. Sites with 355 known Bcbva occurrence are indicated in red. Detection of Bcbva in Taï National Park, Dja Reserve, Dzanga-Sangha Protected Areas and Luebo has been described in previous 
357 studies. For all $B c b v a$ sites, except Luebo, samples were available. Within this study we could identify Grebo as a new site of $B c b v a$ occurrence. $B c b v a$ was not detected at the other tested sub-Saharan sites (indicated in black).

Fig. 2. Bcbva cases in Taï National Park. (A) $B c b v a$ positive and negative carcasses. $38 \%$ of the observed wildlife mortality in Taï National Park is due to Bcbva. Bcbva positive carcasses were broadly distributed throughout the research area with no obvious pattern identifiable. GPS data was available for 113 of 204 detected carcasses and not for those detected before 2001. (B) Bcbva positive and negative fly traps. Five percent of all analyzed flies contained Bcbva genetic material. Flies were also caught outside the research area. A systematic snapshot sampling revealed higher prevalence of $B c b v a$ positive fly traps within the research area.

Fig. 3. Phylogenomic tree of Bcbva isolates. Maximum likelihood tree based on chromosomal sequences of Bcbva isolates from TNP (Côte d'Ivoire, $\mathrm{n}=124$ ) and Grebo (Liberia, $n=2$ ). One sequence per host (mammals/flies, two divergent isolates for fly 600) was included and the final alignment of variant sites measured 298bp.Internal branches with bootstrap values lower than 90 are colored in grey. The colored strip represents different host species. The tree was rooted using the heuristic residual mean squared function in TempEst v 1.5. The scale bar is in substitution per chromosomal site.

\section{Fig. 4. Proportions of simulated chimpanzee communities surviving 150 years with} and without presence of anthrax. Shown are results for different community sizes and anthrax being absent (a, blue boxes) or present ( $\mathrm{p}$, red boxes). Bars represent median 
381 estimates and boxes quartiles across a range of simulation models assuming different

382 inter birth intervals and maximum ages. All models summarized here assumed an annual

383 per capita survival rate of 0.96 .

\section{Methods}

\section{Study sites}

387 TNP covers an area of $3,300 \mathrm{~km}^{2}$ and an additional $200 \mathrm{~km}^{2}$ buffer zone. Since $2001 \mathrm{a}$ 388 veterinary program conducts outbreak investigations in wildlife. We defined the research 389 area as the habitat ranges of the three habituated chimpanzee groups plus a $500 \mathrm{~m}$ buffer 390 zone (103 km²; Fig. S2).

391 Samples belonging to the large-scale data set were collected at 16 sites in 11 sub-Saharan 392 countries stretching from Senegal to Uganda (Fig. S3, Table S1). Most sites (14 out of 393 16) were temporary research sites of the Pan African Programme 394 (www.panafrican.eva.mpg.de) where $B c b v a$ has not been described. Additional samples 395 were obtained from Dja Faunal Reserve (DJR), Cameroon ${ }^{18}$ and Dzanga-Sangha

396 Protected Areas (DSPA), Central African Republic ${ }^{17}$, where $B c b v a$ cases have been 397 previously described. Study sites are described in detail in Supplementary information 398 S1.

\section{$399 \quad$ Necropsies}

400 Carcass monitoring was performed in TNP by a veterinarian, performing necropsies on 401 every carcass reported by researchers working in the forest $(n=173)$. Samples of all inner 402 organs were collected, as far as carcass decomposition allowed. Necropsies followed a 
403 standardized protocol, including use of full personal protective equipment. Carcass sites

404 were decontaminated according to World Health Organization (WHO) guidelines ${ }^{5,28}$. For

405 each sample aliquots were stored in liquid nitrogen and formalin in the field. Frozen

406 samples were transported on dry ice and subsequently stored at $-80^{\circ} \mathrm{C}$. We received

407 additional tissue samples from carcasses sampled by the WHO in TNP between 1996 and

$4082000(n=31)($ Table S2).

409 Rather than using serology, which would also detect animals that survived non-lethal

410 infections, we used PCRs to detect the presence of anthrax in internal organs to confirm

411 that anthrax was the likely cause of death. DNA was extracted from various tissues per

412 animal (liver, spleen and lung when available) using the DNeasy Blood and Tissue Kit

413 (Qiagen, Hilden, Germany); extracts were quantified using a Nanodrop (Thermo Fisher

414 Scientific, Waltham, MA, USA) and stored at $-20^{\circ} \mathrm{C}$. Two hundred ng DNA or $5 \mu 1$ of

415 DNA extract (if DNA concentration was below $40 \mathrm{ng} / \mu \mathrm{l}$ ) were tested for anthrax in

416 duplicate real-time PCR reactions (details in Supplementary Methods S2c). The full

417 anthrax assay used includes three real-time PCRs, each targeting one of the following

418 gene markers: pag (gene for protective antigen) located on the $\mathrm{pXO1}$ plasmid ${ }^{29}$, capB

419 (gene for capsule synthesis) located on pXO2 and Island IV, a chromosomal marker

420 specific for $B c b v a^{15,17}$ (Table S3). Samples were first tested for pag and samples positive

421 in duplicate for pag were tested for capB and Island IV (Extended Data Fig. S1 and

422 Extended Data Fig. S2).

423 Culture under BSL3 conditions was attempted for all PCR positive necropsy samples

424 collected until the end of 2013 (June 2014 for duikers) (Table S2). A native and heat-

425 treated $\left(65^{\circ} \mathrm{C}\right.$ for $30 \mathrm{~min}$, to assess presence of spores) aliquot were plated onto the 
426 following agar plates: Columbia blood agar (Oxoid, Wesel, Germany), blood-

427 trimethoprim agar (1.6 mg trimethoprim, $6.4 \mathrm{mg}$ sulfamethoxazole, $20 \mathrm{mg}$ polymyxin $\mathrm{B}$

428 per liter agar medium) and Cereus Ident agar (Heipha Diagnostica, Eppelheim, Germany)

429 with the chromogenic substrate 5-bromo-4-chloro-3-indoxyl-myoinositol-1-phosphate ${ }^{30}$.

430 Cultures were incubated at $37^{\circ} \mathrm{C}$ and monitored daily. Morphologically suspicious

431 colonies were sub-cultured and tested in real-time PCR. Bcbva was cultured from native

432 and heat-treated samples indicating the presence of heat-resistant spores. Isolates were

433 frozen in Microbank tubes (Mast Diagnostica, Reinfeld, Germany) at $-80^{\circ} \mathrm{C}$.

434 Histopathology was performed on a subset of necropsy samples, including 15 Bcbva PCR

435 positive necropsy samples (Table S2). No signs of anthrax infection were detected in

436 carcasses that were PCR negative for anthrax, while for PCR positive carcasses the most

437 consistent histopathologic finding was per-acute to acute anthrax related pneumonia

438 characterized by mild lymphohistiocytic infiltrates and intraalveolar eosinophilic and

439 proteinaceous or fibrinous material. Numerous bacilli were found intravascular and

440 intraalveolar. Multifocal alveolar and peribronchiolar hemorrhages were present in all

441 animals. Lymph node changes consisted of sinus histiocytosis, cortical hemorrages and

442 edema especially in the mediastinal, tracheobronchiolar and mesenteric lymph nodes.

443 Huge amounts of bacilli were demonstrable in the sinusoids. Within the abdominal cavity

444 the spleen was the organ most affected, with myriads of bacilli visible in the splenic

445 sinusoids, partly embedded in fibrin deposition. There was moderate lymphoid depletion,

446 lymphocytolysis and histiocytosis. The liver parenchyma was severely congested with

447 masses of bacilli within the hepatic sinusoids. All anthrax PCR positive carcasses were 
448 also tested for filoviruses ${ }^{31}$ and respiratory diseases ${ }^{26}$ to rule out co-infection with other

449 common causes of death in this ecosystem.

Blow flies

451 Flies were caught on the ground and in the canopy using custom-made traps (Fig. S4 and 452 S5, Supplementary information S3a). Trapping was done for 60 min or until 20 flies were 453 collected. Flies were euthanized with ether and stored at $-20^{\circ} \mathrm{C}$ in $2 \mathrm{ml}$ Cryotubes (Carl 454 Roth) containing up to 10 flies or at ambient temperature on silica in $50 \mathrm{ml}$ Falcon tubes

455 (Thermo Fisher Scientific) containing up to 20 flies. In TNP, 726 flies were randomly 456 collected within the research area in 2008, 2009, 2012 and 2013 (Table S4). Another 908

457 flies were collected over 19 days in May and June 2014 according to a $2 \times 2 \mathrm{~km}$ grid 458 system covering the research area and $225 \mathrm{~km}^{2}$ surrounding the research area (referred to 459 as "snapshot flies"; Extended Data Fig. S7, Table S4). At a larger scale, 784 flies were 460 collected at 8 sites within 5 sub-Saharan countries (Pan African Programme) from 2012 461 to 2014 (Table S4) and 305 flies were analyzed from two sub-Saharan sites, DJR (n=105) 462 and DSPA ${ }^{17,18}(\mathrm{n}=200)$ (Table S4). In total, 2,723 flies were analyzed (Table S4).

463 DNA extraction of individual flies was performed using the GeneMATRIX Stool DNA 464 Purification Kit (Roboklon, Berlin, Germany). We followed manufacturer's instructions 465 except that each fly was first cut into small pieces using sterilized scissors before being 466 homogenized using a Fast Prep ${ }^{\circledR}$ (MP Biomedicals, Santa Ana, CA, USA). DNA 467 concentration measurements and anthrax testing by real-time PCR were performed as 468 described for necropsy samples (Table S4).

469 A subset of 50 flies containing high pag copy numbers underwent bacterial culture (Table 470 S4, Additional Data Table S1). Half of the fly mush remaining after DNA extraction was 
471 plated directly onto the same culture media described for necropsy samples. Additionally, 472 a $10 \mu \mathrm{l}$ aliquot of the mush was diluted $1: 10$ in sterile $\mathrm{NaCl}$, heat treated for $30 \mathrm{~min}$ at $47365^{\circ} \mathrm{C}$ and plated. $B c b v a$ was retrieved from native and heat-treated samples, indicating 474 the presence of heat-resistant spores in flies. An on-site study in TNP also used direct 475 culture of 204 flies without preceding PCR testing. Flies were homogenized and plated 476 directly onto Cereus Ident agar. Suspicious colonies were sub-cultured on blood477 trimethoprim agar and tested in real-time PCR. This approach yielded another $21 B c b v a$ 478 isolates.

479 To examine whether certain mammals were preferentially affected by $B c b v a$, we tested 480 for differences in fly meal composition of anthrax positive and negative flies. We 481 screened a subset of 750 TNP flies for mammalian DNA using a real time PCR targeting 482 a $130 \mathrm{bp}$ fragment of mammalian 16S mitochondrial DNA (described in Calvignac483 Spencer et al. $\left.{ }^{19}\right)$. We chose a subset of mammal and anthrax positive $(n=28)$ and the 484 according number of mammal positive but anthrax negative flies $(n=29)$ from the same 485 traps (Additional Data Table S1). To dissect fly meal composition, we used a 486 metabarcoding approach, whereby 16S amplicons were deep-sequenced, adapting the 487 amplicon preparation protocol provided by Illumina (San Diego, CA, US)

488 (Supplementary information S3e). We used a custom pipeline to determine taxonomic 489 assessment of each read to the genus and order level described in the Supplementary 490 information S3e (Table S5, Additional Data Tables S1 and S2). Sequences assigned to 491 domestic animals were regarded as contamination as it was shown that even stringent 492 anti-contamination procedures do not prevent the amplification of human and domestic 493 animal sequences present in the environment and reagents ${ }^{32}$. 
494 Details on blow fly analyses and results are in Supplementary information S3.

495 Bones

496 Bones were collected in TNP and 12 Pan African Programme sites in 9 countries (Fig.

497 S3, Table S1 and S7). Bones were transported and stored at ambient temperature. DNA

498 was extracted using a silica-based method ${ }^{33,34}$ (Suppementary information S4b). Bone

499 extracts were tested by real-time PCR as described for necropsy samples (Table S1 and

500 S7). Powder from PCR positive bones was also used for bacterial culture attempts after

501 homogenization in sterile $\mathrm{NaCl}$ (Table S7). We processed the homogenates as described

502 above for necropsy samples with one native aliquot and one heat- treated aliquot. Details

503 on bone analyses and additional results are in Supplementary information S4.

\section{Whole-genome sequencing of Bcbva isolates and SNP calling}

505 Table S8 contains a complete list of all Bcbva isolates sequenced (Fig. S6). Isolate

506 preparation and extraction is described in the Supplementary Methods S6a. Libraries for

507 whole-genome sequencing were prepared with the Nextera XT DNA Library Preparation

508 Kit (Illumina). Libraries were pooled and sequenced on the HiSeq 1500 platform

509 (Illumina) in rapid run mode using either v1 (2x150 bp) or v2 (2x250 bp) chemistry.

$510 \quad$ Illumina adapters were removed using scythe $\mathrm{v} 0.993^{35}$ and trimmed with sickle v1.33

511 applying a quality threshold of 25. Quality trimmed reads were aligned to the reference

512 genome (Bcbva strain CI, Accession numbers CP001746-749) with the BWA-MEM

513 algorithm implemented in $b w a$ v0.7.12-r $1039^{37}$. For conversion to bam format, sorting,

514 deduplication and indexing of aligned reads, we used the picard tools $1.136^{31}$ software

515 package applying the commands SortSam, MarkDuplicates and BuildBamIndex.

516 Subsequent variant calling was performed using the Genome Analysis Toolkit (GATK) 
$517 \quad \mathrm{v} 3.4^{38-40}$. We realigned bam files with the tools RealignerTargetCreator and

518 IndelRealigner. Variants were called with UnifiedGenotyper with a minimum phred

519 scaled confidence threshold of 30 for SNPs to be called. Hard filtering of SNP sites was

520 done with the VariantFiltration command using recommended filter settings. With the

521 SelectVariants command, only SNP sites that passed the filter were selected for further

522 processing. SelectVariants was also used to exclude all SNPs with a coverage $<5 \mathrm{x}$, a

523 minor allele frequency of $>0.1$ and a $G A T K$ Genotype Quality value $<99$. Final

524 consensus sequences were composed with FastaAlternateReferenceMaker. We assessed

525 coverages of all samples with the GATK tools DepthOfCoverage and

526 CoveredByNSamplesSites. Details and further analysis of whole-genome sequencing of

$527 \quad B c b v a$ isolates and SNP calling is Supplementary information S6.

\section{Phylogenetic analyses}

529126 genome sequences (one isolate per mammal/fly) from TNP and GNF (Table S8)

530 were aligned and stripped of non-variant sites with Geneious Pro v8.1.3 (Biomatters

531 ltd. $)^{41}$. Resulting alignments of variable sites were 298, 18 and $11 \mathrm{bp}$ long for the

532 chromosome, $\mathrm{pXO} 1$ and $\mathrm{pXO} 2$ respectively. Given the low number of variable sites in

533 pXO1 and pXO2, we only performed phylogenetic analyses on the chromosome

534 alignment. jModelTest v2.1.4 ${ }^{42}$ was used for determination of the best nucleotide

535 substitution model in a maximum likelihood (ML) framework, resulting in the choice of

536 TVMef ${ }^{43}$.

537 ML analysis was performed with $P h y M L$ v20131022 $2^{44}$ using a combination of subtree-

538 pruning-regrafting (SPR) and nearest-neighbor-interchange (NNI) tree search algorithms.

539 Branch support was estimated using non-parametric bootstrapping with 100 pseudo- 
540 replicates. The tree was rooted using the heuristic residual mean squared function in

541 TempEst $v 1.5^{45}$, placing the root at the position resulting in the most clock-like structure 542 of the data(Fig. 3).

543 We also performed phylogenetic analyses using the Bayesian Markov Chain Monte Carlo 544 (BMCMC) sampling approach implemented in BEAST v1.8.2 $2^{46}$ specifying a constant 545 population coalescent tree prior and assuming an uncorrelated lognormal relaxed 546 molecular clock ${ }^{47}$ (Supplementary information S7c). The maximum clade credibility tree 547 derived from this analysis was very similar to the ML tree (Fig. 3).

548 Another data set was assembled to compare Bcbva from TNP to other strains from sub-

549 Saharan Africa. It included the chromosomal sequences from a representative TNP

550 genome, GNF ones, as well as previously published genomes determined from isolates

551 derived from Bcbva cases in CAR and $\mathrm{CM}^{15,17}$ (Extended Data Fig. S6). The alignment

552 was compiled as described above and contained 1,016 variable positions. Model selection 553 with jModelTest $\mathrm{v} 2.1 .4^{42}$ selected a TPM $1^{48}$ nucleotide substitution model. We performed $554 \quad$ ML analyses as described above.

\section{Statistical analyses}

556 To test the effect of season on the probability of a carcass or fly, respectively, being 557 anthrax positive, we used a Generalized Linear Mixed Model (GLMM) ${ }^{49}$ with binomial 558 error structure and logit link function ${ }^{50}$. As predictors we included the species (monkeys, 559 chimps, duikers, others, blow flies), season and their interaction. 'Season' was modelled 560 by first turning the sampling date into a circular variable and including its sine and cosine 561 into the model. As random intercept effects we included trap id (i.e., GPS location) and 562 the combination of sampling date and GPS location, the latter accounting for potential 
non-independence of flies sampled on the same day from the same trap. We further included random slopes of season within trap id ${ }^{51,52}$. To test the effect of season we compared the full model with a null model lacking the fixed effects of season and its interaction with species ${ }^{53}$, using a likelihood ratio test ${ }^{54}$. Sample size for this model was 1803 samples (carcasses and flies), collected at 352 locations and 328 combinations of sampling date and location including necropsy samples and flies.

In a second model we tested whether the probability of a fly to be tested anthrax positive was influenced by season and the amount of mammalian DNA within in the fly. We used a Generalized Linear Mixed Model (GLMM) ${ }^{49}$ with binomial error structure and logit link function ${ }^{50}$. Into this we included the amount of mammalian DNA found within the fly (determined with real time PCR described above) and season as fixed effects. 'Season' we modeled by first turning the sampling date into a circular variable and then including it sine and cosine into the model. Since the amount of mammalian DNA within the fly was highly skewed, we log transformed it before fitting the model. As random effects (random intercepts) we included the ID of the trap and the date of sampling. To avoid overconfident estimates we included random slopes of the amount of mammal DNA within trap ID and trapping date ${ }^{51,52}$. As an overall test of the effects of the amount of mammal DNA and season we compared the full model with a null model lacking these effects $^{53}$ using a likelihood ratio test ${ }^{54}$. We also used likelihood ratio tests to test for the individual predictors (comparing the full model with a respective reduced model lacking the predictor to be tested ${ }^{51}$ ). We fitted the model in $R^{55}$ using the function glmer of the $R$ package lme4 (version 1.1-10 56 ). To estimate model stability we excluded levels of the random effects one at a time which did not indicate influential levels to exist. The total 
sample size for this model was a total of 474 flies caught on 43 days in 33 traps. (Extended Data Fig. S4, Table S10, Supplementary information S8b).

To evaluate the reproducibility of fly meal identification for each fly we correlated the proportion of sequence counts per amplicon (two amplicons per fly) that was assigned to different mammalian genera using Spearman correlation. To test whether there were differences in fly meal composition of anthrax positive and anthrax negative flies, we tested whether detection of a given mammal taxon in a fly sample was associated with anthrax positivity. We used GLMMs ${ }^{49}$ applied separately for each mammal genus identified in the flies. The response was whether the fly was anthrax positive and the key predictor with fixed effect was mammal presence. We considered a mammal to be present when it was detected in at least one of the two amplicons per fly. We included only those mammal genera in the model that were detected in at least five of all generated amplicons (two per fly). In addition to mammal presence, we included tid and the factor sampling date as random effects (random intercepts) ${ }^{51,52}$. Models were fitted with binomial error structure and logit link function ${ }^{50}$. Sample size for all models was 57 flies, caught in 22 different traps on 13 days. To test whether mammal presence had an impact on anthrax positivity, we dropped mammal presence from the model ${ }^{53}$ and compared the models using a likelihood ratio test ${ }^{54}$. Model stability was accessed as above. We fitted models at two different taxonomic resolutions: one with taxonomic assignment at genus level and the other at order level. GLMMs were fit in $R^{55}$ using the function glmer of the $R$ package $\operatorname{lme} 4 \mathrm{v} 1.1-10^{56}$

To evaluate geographic distribution of $B c b v a$ in TNP we checked whether, due to higher mammal density ${ }^{23}, B c b v a$ was more likely to occur inside the research area. To test this 
609 hypothesis we analyzed 908 flies from 83 different traps (Extended Data Fig. S7, Table

610 S4, Additional Data Table S1). 21 traps were located within the research area and 62

611 traps in the adjoining forest belt. 8/21 traps within the research area were anthrax positive

612 and 8/62 outside the research area. We compared the two groups using Fisher Exact's

613 Test (Supplementary information S8d).

614 To learn more about the spatial dynamics of $B c b v a$ in TNP, we investigated the

615 correlation between genetic and geographic distances. To correct for genetic and spatial

616 autocorrelation, we excluded strains from the data set that originated from the same fly

617 catching point (in a $1 \mathrm{~km}^{2}$ radius) on the same day or from the same followed-up outbreak

618 in mammals. Only one strain was kept per outbreak or fly catching point, the selection

619 criterion being high average coverage of the genome (Table S8, Supplementary

620 information S8e). Geographic distances (in $\mathrm{km}$ ) were derived from GPS data using

621 GeographicDistanceMatrixGenerator $\mathrm{v} 1 \cdot 2 \cdot 3^{57}$. Genetic distances were approximated

622 using the relative distances drawn from a Maximum Likelihood Tree built in PhyML

$623 v 20131022^{44}$ with the $R$ package ape ${ }^{58}$ using the cophenetic function. Multiple regression

624 on distance matrices (MRM) as implemented in the R ecodist package ${ }^{59}$ using 1000

625 permutations and Spearman correlations was performed on genetic and geographic

626 distance matrices. To examine variation within genetic lineages, we binned our data by

627 genetic distance (bin size=relative genetic distance of 0.03, approx. 2.5 SNPs) and

628 focused on groups with low genetic distance (max relative genetic distance $<0.5)$ and

629 their mean geographic distance (Extended Data Fig. S9). Homogeneity of variance

630 between groups was assured with the Fligner Killeen test $(\mathrm{p}=0.07 ;>0.05$ as requested). 
631 To evaluate the impact Bcbva could have on the TNP chimpanzee population, we 632 conducted a simulation (Supplementary information S8f). We first defined a series of 633 population parameters for the simulation ${ }^{27}$. We simulated the survival prospects of 634 chimpanzee communities of a given size, with individuals reproducing at certain regular 635 intervals after maturation, having a maximum age, and an annual survival probability. 636 Since most of these parameters are associated with considerable uncertainty and since we 637 wanted to assess to what extent the simulation results depend on the particular parameters 638 chosen we parameterized the simulations as follows: Initial community size: 20 to 80 639 individuals (increment: 10); inter-birth interval: 4 to 7 years (increment: 1); interval after 640 death of infant: 1 year; maximum age: 40 to 50 years (increment: 2); age of first 641 reproduction of males and females: 10 and 14 years, respectively. Since per capita annual 642 survival probability without the influence of anthrax is unknown (mortality cases due to 643 anthrax may not be detected in all cases, in particular before necropsies were made 644 systematically), we simulated per capita annual survival probabilities from 0.93 to 0.99 645 (increment: 0.03 ). In addition, we made survival probability density dependent, as this is 646 a common characteristic observed in many species including chimpanzees ${ }^{60}$. For this we 647 introduced a logistic function $(1 /(1+\exp (-(20-0.08 *$ community size $))))$ that increased or 648 reduced mortality rate as a function of chimpanzee community size. At the beginning of 649 each simulation run we generated a community of the simulated size by randomly 650 allocating sexes (proportion of females: 0.7) and ages (uniformly distributed between 10 651 and the simulated maximum age) to individuals. To avoid stochastic effects of the 652 initially generated community, we let the simulation run for 50 time steps (i.e., 'years') 653 without anthrax presence before the evaluated time period began. 
654 We estimated the risk of annual anthrax outbreak probability, its dependence on

655 community size and the number of individuals affected as exp $(-1.83+0.039 *$ community

656 size) from a Poisson regression (null, full model comparison, $\chi^{2}=7.89, \mathrm{df}=1, \mathrm{p}<0.01$ ).

657 We simulated both an anthrax and a non-anthrax scenarios for 150 time steps (i.e., 'years')

658 with 100 replications each and for each possible combination of the simulated

659 parameters. Communities were considered to be extinct, when no reproducing females

660 were present.

661 All $R$ scripts are available upon request. Details on statistical analyses and additional

662 results are in Supplementary information S8.

\section{Data availability}

664 Raw reads of 16S amplicons are available in the European Nucleotide Archive (ENA)

665 under project accession number PRJEB14554, sample accession numbers ERS1217219-

666 336. Raw reads for all 178 Bcbva isolates from TNP and GNF are available in the ENA

667 under project accession number PRJEB14616, sample accession numbers ERS1222903-

668 3080. Variable position alignments are available from the Dryad Digital Repository:

669 http://dx.doi.org/10.5061/dryad.v8bn7.

670 


\section{$671 \quad$ Extended data figure legends}

672 Extended Data Fig. S1. Necropsies performed since 1996. Shown is the total amount

673 of necropsies performed per year in TNP from 1996 to 2015. Grey bars indicate the

674 number and according proportion of Bcbva positive necropsies. In the years 2003 and

6752010 only limited veterinary service was available at TNP due to political insecurity in

676 the region.

677

678

679

680

681

682

683

684

685

686

687

688

689

690

691

692

693

\section{Extended Data Fig. S2. Geographic location of Bcbva positive carcasses in Taï}

National Park. Shown are Bcbva positive tested necropsies in TNP since 2001. GPS data was available for 70 of all positive tested $(n=81)$ necropsies.

\section{Extended Data Fig. S3. Effect of mammalian DNA content on anthrax positivity in}

flies. . Shown is the probability of $B c b v a$ positivity (PA, pag respectively) as a function of the amount of mammalian DNA (copies) found in a fly. The amount of mammal DNA was binned (bin width 0.25 ) and the area of the points depicts the number of flies (range: 1 to 206) in the respective bins. The dashed line indicates the fitted model and the dotted lines the $95 \%$ confidence interval.

\section{Extended Data Fig. S4. Effect of season on anthrax positivity in flies. Shown is the} probability of $B c b v a$ (PA) positivity over the course of a year (binned in 10 day periods). The area of the points depicts the number of flies in the respective ten days period. The dashed line indicates the fitted model and the dotted lines the $95 \%$ confidence interval. 


\section{Extended Data Fig. S 5. Maximum clade credibility tree based on chromosomal} sequences of Bcbva isolates from TNP (Côte d'Ivoire, n=124) and Grebo (Liberia, $\mathbf{n = 2 ) . ~ O n e ~ s e q u e n c e ~ p e r ~ h o s t ~ h o s t s ~ ( m a m m a l s / f l i e s , ~ t w o ~ d i v e r g e n t ~ i s o l a t e s ~ f o r ~ f l y ~ 6 0 0 ) ~}$ was included and the final alignment of variant sites measured 298bp. Size of nodes represents posterior probability values. The location of the root received a posterior probability of 1 .

\section{Extended Data Fig. S 6. Maximum likelihood tree for sub-Saharan Bcbva strains.}

ML tree based on chromosomal sequences of $B c b v a$ strains from Côte d'Ivoire, Cameroon, Central African Republic and Liberia. The alignment of variant sites measured 1016bp. Bootstrap values are shown above branches and the scale bar reflects the genome-wide substitution rate. The tree was rooted using TempEst v 1.5.

Extended Data Fig. S 7. Fly snapshot sampling scheme. For the fly snapshot flies were caught following a 2x2 km grid system within and outside the research area within 19 days. In total 908 snapshot flies were analyzed.

\section{Extended Data Fig. S 8. Genetic and geographic distances of Bcbva isolates from the} fly snapshot. (A) Maximum Likelihood Tree based on chromosomal sequences of $B c b v a$ isolates from the 19 day fly snapshot. Each dot represents one fly isolate. Colors were chosen to illustrate the distribution of genetically clustering isolates on the map presented in panel B. The final alignment of variant sites measured 123bp. Bootstrap values are shown above all internal branches. The tree was rooted using the "best-fit" option in 
717 Path-O-Gen v1.2. The scale bar is in substitution per site. (B) Geographic origin of Bcbva

718 isolates collected during the fly snapshot. Colors correspond to ML tree (A). Big circles

719 represent two isolates.

720

721 Extended Data Fig. S 9. Boxplot of genetic and mean geographic distances. Bcbva

722 isolates from TNP were binned by relative genetic distance (bin size $=0.03$, approx. 2.5

723 SNPs). The two most genetically distant isolates received a value of 1 and all other

724 distances were scaled accordingly. Diamonds indicate the geographic distance means of

725 the groups. To examine variation within genetic lineages, we analyzed isolates with low

726 genetic distance (max relative genetic distance $<0.5$, marked in blue) and their mean

727 geographic distance. For low genomic distances, genetic and mean geographic distances

728 are correlated $(\mathrm{p}=4 \times 10-5, \mathrm{R} 2=0.72)$.

729

730 Extended Data Fig. S10. Fly species composition based on GMYC analysis. Fly

731 species composition for three sites with known Bcbva occurrence: TNP (Côte d'Ivoire)

732 (A), DJR (Cameroon) (B), DSPA (Central African Republic) (C). Shown are the

733 proportions of flies per site in \% belonging to one single fly species identified with

734 GMYC models. Different colors indicate different taxonomic fly families. 


\section{References (28-60)}

28 Turnbull, P. C. B. (World Health Organization, Department of Communicable Diseases Surveillance and Response, 1998).

29 Ellerbrok, H. et al. Rapid and sensitive identification of pathogenic and apathogenic Bacillus anthracis by real-time PCR. FEMS Microbiol Lett 214, 5159 (2002).

30 Klee, S. R. et al. Characterization of Bacillus anthracis-like bacteria isolated from wild great apes from Cote d'Ivoire and Cameroon. J Bacteriol 188, 5333-5344 (2006).

31 Panning, M. et al. Diagnostic reverse-transcription polymerase chain reaction kit for filoviruses based on the strain collections of all European biosafety level 4 laboratories. Journal of Infectious Diseases 196, S199-S204 (2007).

32 Leonard, J. A. et al. Animal DNA in PCR reagents plagues ancient DNA research. Journal of Archaeological Science 34, 1361-1366 (2007).

33 Gamba, C. et al. Comparing the performance of three ancient DNA extraction methods for high-throughput sequencing. Molecular ecology resources 16, 459469, doi:10.1111/1755-0998.12470 (2016).

34 Rohland, N. \& Hofreiter, M. Ancient DNA extraction from bones and teeth. Nature protocols 2, 1756-1762, doi:10.1038/nprot.2007.247 (2007).

35 Buffalo, V. Scythe: A 3'-end adapter contaminant trimmer [Software]. Available at https://github.com/vsbuffalo/scythe.

36 Joshi, N. A. \& Fass, J. N. Sickle: A sliding-window, adaptive, quality-based trimming tool for FastQ files [Software]. Available at https://github.com/najoshi/sickle. (2011).

37 Li, H. Aligning sequence reads, clone sequences and assembly contigs with BWA-MEM. arXiv preprint arXiv:1303.3997 (2013).

38 Auwera, G. A. et al. From FastQ data to high-confidence variant calls: the genome analysis toolkit best practices pipeline. Current Protocols in Bioinformatics, 11.10. 11-11.10. 33 (2013).

39 DePristo, M. A. et al. A framework for variation discovery and genotyping using next-generation DNA sequencing data. Nature genetics 43, 491-498 (2011).

40 McKenna, A. et al. The Genome Analysis Toolkit: a MapReduce framework for analyzing next-generation DNA sequencing data. Genome Res 20, 1297-1303, doi:10.1101/gr.107524.110 (2010).

41 Kearse, M. et al. Geneious Basic: an integrated and extendable desktop software platform for the organization and analysis of sequence data. Bioinformatics 28, 1647-1649 (2012).

42 Darriba, D., Taboada, G. L., Doallo, R. \& Posada, D. jModelTest 2: more models, new heuristics and parallel computing. Nat Methods 9, 772, doi:10.1038/nmeth.2109 (2012).

43 Posada, D. Using MODELTEST and PAUP* to select a model of nucleotide substitution. Current protocols in bioinformatics, 6.5. 1-6.5. 14 (2003).

44 Guindon, S. et al. New algorithms and methods to estimate maximum-likelihood phylogenies: assessing the performance of PhyML 3.0. Syst Biol 59, 307-321, doi:10.1093/sysbio/syq010 (2010). 
45 Rambaut, A., Lam, T. T., Max Carvalho, L. \& Pybus, O. G. Exploring the temporal structure of heterochronous sequences using TempEst (formerly Path-OGen). Virus Evolution 2 (2016).

46 Drummond, A. J., Suchard, M. A., Xie, D. \& Rambaut, A. Bayesian phylogenetics with BEAUti and the BEAST 1.7. Mol Biol Evol 29, 1969-1973, doi:10.1093/molbev/mss075 (2012).

47 Drummond, A. J., Ho, S. Y., Phillips, M. J. \& Rambaut, A. Relaxed phylogenetics and dating with confidence. PLoS biology 4, e88, doi:10.1371/journal.pbio.0040088 (2006).

48 Kimura, M. Estimation of evolutionary distances between homologous nucleotide sequences. Proceedings of the National Academy of Sciences 78, 454-458 (1981).

49 Baayen, R. H. Analyzing linguistic data: A practical introduction to statistics using R. (Cambridge University Press, 2008).

50 McCullagh, P. \& Nelder, J. A. Generalized linear models. Vol. 37 (CRC press, 1989).

51 Barr, D. J., Levy, R., Scheepers, C. \& Tily, H. J. Random effects structure for confirmatory hypothesis testing: Keep it maximal. J Mem Lang 68, doi:10.1016/j.jml.2012.11.001 (2013).

52 Schielzeth, H. \& Forstmeier, W. Conclusions beyond support: overconfident estimates in mixed models. Behavioral Ecology 20, 416-420 (2009).

53 Forstmeier, W. \& Schielzeth, H. Cryptic multiple hypotheses testing in linear models: overestimated effect sizes and the winner's curse. Behavioral Ecology and Sociobiology 65, 47-55 (2011).

54 Dobson, A. J. \& Barnett, A. An introduction to generalized linear models. (CRC press, 2008).

55 Team, R. C. R: A language and environment for statistical computing., (R Foundation for Statistical Computing, Vienna, Austria, 2015).

56 Bates, D., Mächler, M., Bolker, B. \& Walker, S. Fitting linear mixed-effects models using lme4. Journal of Statistical Software 67, 1-48 (2014).

57 Ersts, P. Geographic distance matrix generator (version 1.2. 3). American Museum of Natural History. Available at http://biodiversityinformatics.amnh.org/open_source/gdmg. Center for Biodiversity and Conservation (2011).

58 Paradis, E., Claude, J. \& Strimmer, K. APE: Analyses of Phylogenetics and Evolution in R language. Bioinformatics 20, 289-290 (2004).

59 Goslee, S. C. \& Urban, D. L. The ecodist package for dissimilarity-based analysis of ecological data. Journal of Statistical Software 22, 1-19 (2007).

60 Kuehl, H., Elzner, C., Möbius, Y., Boesch, C. \& Walsh, P. D. The price of play: Self-organized infant mortality cycles in chimpanzees. PLoS One 3, doi:10.1371/journal.pone.0002440 (2008). 